\title{
Effects of Parental Weight and \\ Fatness on Relative Weight and \\ Fatness of Young Children \\ Comparison of Cross-Sectional and \\ Prospective Clinical Data ${ }^{a}$
}

\author{
JOEL A. GRINKER, ${ }^{b}$ STANLEY M. GARN, ${ }^{c}$ \\ AND KAREN R. ROSENBERG ${ }^{d}$ \\ ${ }^{b}$ Human Nutrition \\ School of Public Health \\ Department of Pediatrics \\ University of Michigan \\ Ann Arbor, Michigan 48109 \\ ${ }^{c}$ Human Nutrition \\ School of Public Health \\ Center for Human Growth and Development \\ University of Michigan \\ Ann Arbor, Michigan 48109 \\ ${ }^{d}$ Department of Anthropology \\ University of Delaware \\ Newark, Delaware 19716
}

Fatness follows family lines. Twin and adoption studies suggest that genetic factors play a major role in the familial transmission of fatness, ${ }^{1-14}$ although environmental influences are also recognized. ${ }^{5}$ The fatness of young children as a function of the fatness of their mothers is examined in data selected from a cross-sectional epidemiological study (Ten-State Nutrition Survey) and a prospective clinical study. Only data from obese $(\mathrm{O})$ or nonobese white women and children were included.

\section{TEN-STATE DATA}

Anthropometric data (tricep and subscapular skinfolds, weight and height) were available from mothers and children aged 1 to 12 years. Maternal fatness was defined

${ }^{a}$ Supported in part by National Institutes of Health Grant \#AM32944 and Gerber Foods. National Center for Health Statistics data made available by Ken Guire, Dept. of Biostatistics, School of Public Health, University of Michigan, Ann Arbor, MI. 
TABLE 1. Triceps Skinfold of White Children in Ten-State Survey by Fatness ${ }^{a}$ of Their Mothers

\begin{tabular}{|c|c|c|c|c|c|c|c|c|c|}
\hline \multirow{2}{*}{$\begin{array}{l}\text { Age of } \\
\text { Children }\end{array}$} & \multicolumn{3}{|c|}{ Lean Mothers } & \multicolumn{3}{|c|}{ Medium Mothers } & \multicolumn{3}{|c|}{ Obese Mothers } \\
\hline & $n$ & Mean & $\mathrm{SD}$ & $n$ & Mean & $\mathrm{SD}$ & $n$ & Mean & SD \\
\hline \multicolumn{10}{|l|}{ BOYS } \\
\hline $0-1$ & 15 & 8.1 & 3.23 & 61 & 8.9 & 2.97 & 12 & 10.5 & 4.08 \\
\hline $1-2$ & 20 & 8.2 & 3.15 & 94 & 10.8 & 5.67 & 19 & $10.6^{b}$ & 1.95 \\
\hline $2-3$ & 23 & 9.5 & 2.45 & 98 & 9.2 & 2.36 & 24 & 10.5 & 3.05 \\
\hline $3-4$ & 27 & 8.1 & 2.14 & 120 & 9.4 & 2.87 & 23 & $9.9^{b}$ & 2.76 \\
\hline $4-5$ & 30 & 7.9 & 2.52 & 115 & 9.4 & 3.11 & 37 & $10.3^{b}$ & 3.73 \\
\hline $5-6$ & 30 & 8.0 & 2.48 & 139 & 8.5 & 2.63 & 38 & $10.6^{b}$ & 4.59 \\
\hline $6-7$ & 33 & 7.9 & 2.85 & 150 & 8.7 & 3.13 & 38 & 8.8 & 2.98 \\
\hline $7-8$ & 42 & 7.7 & 2.74 & 156 & 8.6 & 3.51 & 48 & $10.0^{b}$ & 3.59 \\
\hline $8-9$ & 42 & 8.8 & 3.68 & 154 & 9.9 & 5.32 & 27 & $11.4^{b}$ & 5.43 \\
\hline $9-10$ & 25 & 9.3 & 3.93 & 148 & 9.9 & 5.25 & 43 & $12.9^{b}$ & 7.40 \\
\hline $10-11$ & 33 & 8.8 & 3.97 & 164 & 11.3 & 5.38 & 39 & $13.7^{b}$ & 6.42 \\
\hline $11-12$ & 27 & 9.8 & 3.99 & 168 & 11.9 & 6.38 & 45 & $14.4^{b}$ & 7.66 \\
\hline \multicolumn{10}{|l|}{ GIRLS } \\
\hline $0-1$ & 19 & 8.1 & 2.65 & 80 & 10.0 & 9.19 & 15 & 9.8 & 4.47 \\
\hline $1-2$ & 20 & 9.0 & 2.32 & 96 & 10.1 & 2.80 & 17 & 9.2 & 2.73 \\
\hline $2-3$ & 22 & 8.8 & 2.52 & 90 & 9.9 & 2.35 & 26 & 10.2 & 3.73 \\
\hline $3-4$ & 19 & 8.0 & 2.95 & 103 & 10.2 & 2.66 & 24 & $10.4^{b}$ & 3.05 \\
\hline $4-5$ & 31 & 9.0 & 2.96 & 116 & 10.3 & 6.23 & 26 & $10.8^{b}$ & 3.36 \\
\hline $5-6$ & 28 & 8.3 & 3.85 & 133 & 10.2 & 2.98 & 25 & $11.1^{b}$ & 3.57 \\
\hline $6-7$ & 29 & 8.6 & 1.88 & 142 & 9.8 & 3.30 & 45 & $11.5^{b}$ & 3.73 \\
\hline $7-8$ & 41 & 9.0 & 2.98 & 150 & 10.6 & 3.62 & 38 & $11.5^{b}$ & 3.95 \\
\hline $8-9$ & 33 & 10.4 & 5.33 & 146 & 11.5 & 4.18 & 43 & $14.6^{b}$ & 6.50 \\
\hline $9-10$ & 28 & 11.1 & 4.70 & 133 & 12.1 & 4.74 & 39 & $15.9^{b}$ & 8.31 \\
\hline $10-11$ & 27 & 10.8 & 6.11 & 136 & 13.4 & 5.72 & 34 & $16.7^{b}$ & 8.32 \\
\hline $11-12$ & 37 & 11.3 & 4.94 & 146 & 14.1 & 5.85 & 33 & $18.5^{b}$ & 8.65 \\
\hline
\end{tabular}

${ }^{a}$ Fatness of mother is defined with reference to triceps skinfold.

${ }^{b} p<0.05$. Comparison: mean obese versus lean.

in reference to the Z-score for tricep (TRI) skinfolds and reduced to three groups: (1) Lean $=\langle-1 \mathrm{Z}$ score; (2) medium $=-1$ to $1 \mathrm{Z}$ score and (3) obese $=>1 \mathrm{Z}$ score. The children of obese women are systematically heavier than those of lean women by age three (boys) and two (girls) and significantly fatter (TRI) by age four (see TABLE 1). No systematic height differences were found between children of obese or lean mothers. Children of lean and obese mothers have different patterns of fatness $(<$ and $>$ TRI) from those of medium mothers by ages two to three.

\section{MICHIGAN DATA}

Anthropometric data were obtained from healthy middle-income mothers and term healthy infants at delivery and follow-up data (1-48 months) from pediatricians' examinations. Mothers were selected to meet obesity or normal-weight/fatness criteria that were defined on the basis of prepregnancy weight/height standards and postpartum skinfold measures. ${ }^{6}$ Each infant's weight and height were converted to the appropriate percentiles for weight/age, height/age and weight/height using computerized programs based on National Center for Health Statistics values. and normalized $\mathrm{Z}$ scores were 


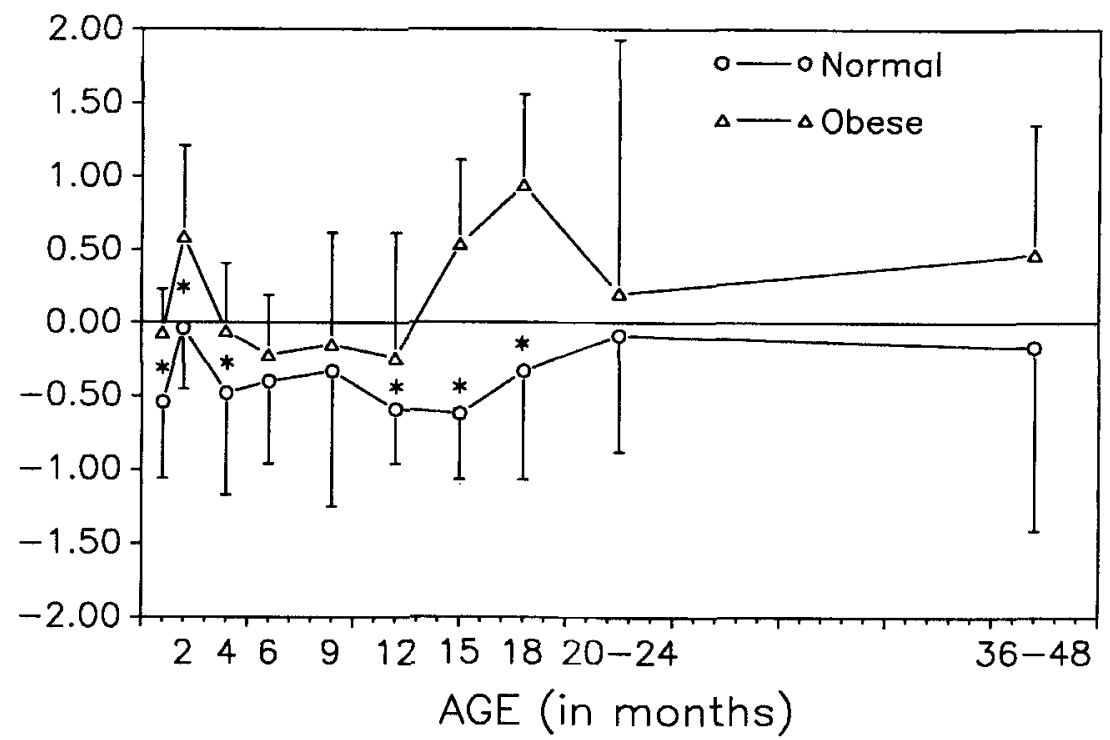

FIGURE 1. Mean (variance) normalized weight/height $Z$ scores for infants from obese and normal-weight/fatness mothers during a longitudinal study from birth to 48 months. Obesity was defined on the basis of $>85$ th percentile height/weight TRI and MAC measurements. Sample sizes at different time points vary as a function of number of infants for whom data were available from pediatricians; $n=17$ obese and 20 medium at one month, 24 and 26 at 2 months, 23 and 21 at 4 months, 20 and 27 at 6 months, 16 and 19 at 9 months, 17 and 20 at 12 months, 12 and 16 at 15 months, 5 and 11 at 18 months, 14 and 14 at 20-24 months, and 15 and 14 at 36-48 months.

derived.' Infants of obese mothers were significantly heavier and fatter at birth and remained heavier than infants of normal-weight/fatness (M) mothers. ${ }^{6.8}$ Infants of obese mothers had consistently larger weight/age $Z$ scores (significantly so at 1,2 , $6,9,12,15,18$, and $36-48$ months) and weight/height $Z$ scores (significantly so at $1,2,4,12,15$, and 18 months) than infants of median mothers (see Fig. 1). Height/age $Z$ scores were not different at any age. Five infants of obese mothers and no infants of medium mothers met the criteria for obesity during the first six months. Combined paternal and maternal obesity produced significantly larger weight/height $\mathrm{Z}$ scores $(2,12,15$, and $20-24$ months) compared with the $Z$ scores of infants of obese only and medium mothers.

Systematic effects of maternal fatness on early childhood fatness were obtained in both studies supporting genetic and environmental explanations in the development of childhood fatness. Results demonstrate that even small sample sizes (19-23 within sex and age groups in the first study and 12-16 in the second study) can illustrate the strong familial transmission of fatness.

\section{REFERENCES}

1. BorJeson, M. 1976. The aetiology of obesity in children. Acta. Paediatr. Scand. 65: 279-287. 
2. Stunkard, A. J., T. T. Foch \& Z. Hrubec. 1986. A twin study of human obesity. J. Am. Med. Assoc. 256: 51-54.

3. Stunkard, A. J., T. I. A. Sorenson, C. Hanis et al. 1986. An adoption study of human obesity. N. Engl. J. Med. 314: 193-198.

4. Price, R. A., R. J. Cadoret, A. J. Stunkard et al. 1987. Genetic contributions to human fatness: An adoption study. Am. J. Psychiatry 144: 1003-1008.

5. Garn, S. M., S. M. Bailey \& P. E. Cole. 1976. Similarities between parents and their adopted children. Am. J. Phys. Anthropol. 45: 539-544.

6. Grinker, J. A. \& K. Bose. 1987. Obesity prospectives: . . . in familial obesity. In Recent Advances in Obesity Research: V. Berry, E. M., S. H. Blondheim, H. E. Eliahou et al., Eds.: 383-390. Libbey \& Co. London.

7. Hamml, P. V. V., T. A. Drizd, C. L. Johnson et al. 1979. Physical growth: National Center for Health Statistics percentiles. Am. J. Clin. Nutr. 32: 607.

8. Grinker, J. A., J. G. Rubin \& K. Bose. 1986. Sweet preference and body fatness. Nutr. Behav. 3: 197-211. 